\title{
DIFFRACTION BY A HALF-PLANE IN A CHIRAL MEDIUM (NORMAL INCIDENCE)
}

\begin{abstract}
S. Przeździecki
Institute of Fundamental Technological Research, Polish Academy of Sciences

Świętokrzyska 21, Warszawa, Poland

(Received February 16, 1993)

An exact, closed form solution is presented for a diffraction problem by a half-plane embedded in a chiral medium. The incidence direction of an electromagnetic plane wave is assumed to be normal to the edge of the half-plane. The problem has been reduced to a boundary value problem for two modal potentials satisfying distinct Helmholtz equations and coupled by the boundary conditions at the half-plane. A solution is constructed with the aid of the Wiener-Hopf technique. A basic feature of the problem is its two-mode character exhibited by: two families of difracted rays, refleciion coupling of the modes, excitation of lateral waves.
\end{abstract}

PACS numbers: 41.20. $-q, 42.25$.Fx

\section{Introduction}

For electromagnetic fields harmonically dependent on time via $\exp (-i \omega t)$ a chiral medium is described by the following constitutive relations [1]:

$$
\boldsymbol{D}=\varepsilon \boldsymbol{E}+\mathrm{i} \chi \boldsymbol{H}, \quad \boldsymbol{B}=\mu \boldsymbol{H}-\mathrm{i} \chi \boldsymbol{E} .
$$

A medium defined by (1.1) is also said to be optically active.

In this paper, an exact, closed form solution is given for the following diffraction problem: a perfectly conducting half-plane is embedded in a chiral medium and scatters an incident electromagnetic plane wave. The direction of incidence is normal to the edge of the half-plane.

Thus the problem is two-dimensional with no dependence on the coordinate directed along the edge.

A basic property of propagation in a chiral medium is that for any direction there exist two plane waves of circular polarizalions propagating with distincl speeds. In more general terms: the two polarization modes of an electromagnetic field are distinguished by different propagation velocities (removal of degeneration characteristic of ordinary, isotropic media). 
The essential aspect of the problem consists in that it reduces to the following boundary value problem for two functions $u, v$ :

1. equations to be obeyed are two distinct Helmholtz equations

$$
\left(\nabla^{2}+k_{1}^{2}\right) u=0, \quad\left(\nabla^{2}+k_{2}^{2}\right) v=0
$$

2. boundary conditions to be fulfilled at the half-plane are

$$
u+v=w_{1}, \quad \frac{\partial}{\partial z}\left(k_{2} u-k_{1} v\right)=w_{2} .
$$

$z$ is a Cartesian coordinate perpendicular to the half-plane, $w_{1}, w_{2}$ are simple exponential functions defined by an incident wave. Moreover, in order to assure uniqueness, $u$ and $v$ are required to behave properly at the edge of the half-plane and at infinity.

The problem (1.2), (1.3), after splitting into its even and odd part with respect to $z$, is solved with the aid of the Wiener-Hopf technique [2]. The kernel function in the Wiener-Hopf equations is of a form encountered earlier [3, 4] and can be factored effectively leading to a simple solution given by quadratures.

Fundamental features of the problem follow from the formulation (1.2), (1.3).

1 . The problem is two-moded (essentially vectorial), since the two polarization modes couple at the half-plane and consequently it cannot be reduced to a boundary value problem for one scalar function satisfying a second-order differential equation (one-mode problem) or be decomposed into independent (uncoupled) one-mode problems (scalarizable problem).

2. The modal coupling is of general type and comprises a reflection coupling, i.e. the coupling that occurs on reflection from an entire perfectly conducting plane. This is in contrast to scalarizable problems with no modal coupling (e.g. electromagnetic, half-plane, the Sommerfeld problem) or to problems where the coupling is exclusively due to edge diffraction without any reflection coupling [5, $6]$.

3. The problem involves an interaction of two distinct wave species; it will be seen that this results in the excitation of lateral waves propagating along the half-plane.

An electromagnetic problem closely analogous to the present one (two wave species, reflection coupling) was solved in [7].

A detailed investigation and interpretation of the solution is postponed to a later paper. In the light of the work done in $[4,8-11]$ this is rather a matter of relevant adaptations. In Sec. 9 we only outline some of the basic properties of the solution, calling attention to interesting phenomena of two families of diffracted rays and of the excitation of lateral waves.

Generalization of the problem considered here to the case of arbitrary (oblique) incidence will be presented in a forthcoming paper [12].

\section{Geometry and basic equations}

We choose a right-handed system of Cartesian coordinates $x, y, z$ in such a way that the diffracting half-plane is determined by $z=0, x \geq 0$ (Fig. 1). We denote by $x, y, z$ the unit vectors directed along the respective axes. 


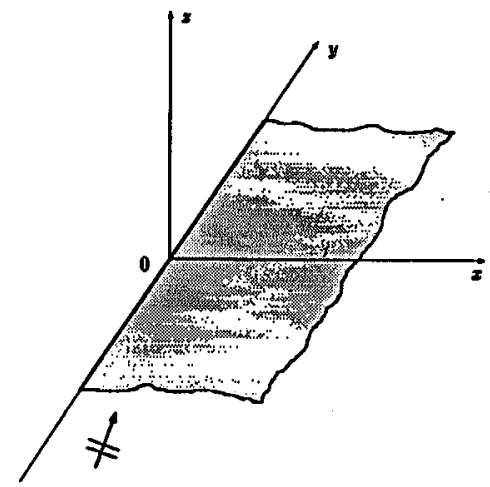

Fig. 1. System of coordinates, the diffracting half-plane and the incident waves.

For a chiral medium (1.1) the Maxwell system takes the form

$$
\boldsymbol{\nabla} \times \boldsymbol{H}=-\mathrm{i} \omega \boldsymbol{E}+\omega \chi \boldsymbol{H}, \quad \boldsymbol{\nabla} \times \boldsymbol{E}=\mathrm{i} \omega \mu \boldsymbol{H}+\omega \chi \boldsymbol{E} .
$$

For fields independent of $y\left(\frac{\partial}{\partial y} \equiv 0\right)$ the components $E_{y}=\phi, I I_{y}=\psi$ may serve as potentials for the remaining planar fields

$$
\boldsymbol{e}=\boldsymbol{E}-\phi \boldsymbol{y}, \quad \boldsymbol{h}=\boldsymbol{H}-\psi \boldsymbol{y} .
$$

By simple algebra we get from (2.1):

$$
\begin{aligned}
& e=\frac{\omega}{\nu} y \times\left(\chi \nabla_{t} \phi-\mathrm{i} \mu \nabla_{t} \psi\right), \\
& h=\frac{\omega}{\nu} y \times\left(\mathrm{i} \varepsilon \nabla_{t} \phi+\chi \nabla_{t} \psi\right),
\end{aligned}
$$

where $\nu=\omega^{2}\left(\varepsilon \mu-\chi^{2}\right), \nabla_{t}=\nabla-y \frac{\partial}{\partial y}$.

It also follows from (2.1) that the components $\phi, \psi$ fulfil the following system of equations:

$$
\left(\begin{array}{cc}
\nabla_{t}^{2}+\omega^{2}\left(\varepsilon \mu+\chi^{2}\right) & 2 \mathrm{i} \omega^{2} \mu \chi \\
-2 \mathrm{i} \omega^{2} \varepsilon \chi & \nabla_{t}^{2}+\omega^{2}\left(\varepsilon \mu+\chi^{2}\right)
\end{array}\right)\left(\begin{array}{l}
\phi \\
\psi
\end{array}\right)=\left(\begin{array}{l}
0 \\
0
\end{array}\right) .
$$

Equations (2.4) are equivalent to (2.1) when $e, h$ are determined via (2.3). Thus any solution to (2.1) can be generated from $\phi, \psi$ obeying (2.4).

From (2.4) we get

$$
\left(\nabla_{t}^{2}+k_{1}^{2}\right)\left(\nabla_{t}^{2}+k_{2}^{2}\right) \phi=0
$$

where

$$
k_{1}=\omega(\sqrt{\varepsilon \mu}+\chi), \quad k_{2}=\omega(\sqrt{\varepsilon \mu}-\chi) .
$$

Consequently (see Appendix)

$$
\phi=u+v
$$

and

$$
\left(\nabla_{t}^{2}+k_{1}^{2}\right) u=0, \quad\left(\nabla_{t}^{2}+k_{2}^{2}\right) v=0
$$


From (2.4) we obtain

$$
\psi=-\mathrm{i} Y(u-v), \quad Y=\sqrt{\varepsilon / \mu} .
$$

Taking into account Eqs. (2.7), (2.8) and (2.3), we can now write for any solution $\boldsymbol{E}, \boldsymbol{H}$ to $(2.1)$ :

$$
\begin{array}{ll}
E=E^{(1)}+E^{(2)}, \quad H=H^{(1)}+H^{(2)}, \\
E^{(1)}=\frac{1}{k_{1}} y \times \nabla_{t} u+u y, & H^{(1)}=-\mathrm{i} Y E^{(1)}, \\
\boldsymbol{E}^{(2)}=\frac{1}{k_{2}} y \times \nabla_{t} v+v y, & \boldsymbol{H}^{(2)}=-\mathrm{i} Y E^{(2)} .
\end{array}
$$

Each of the fields $\boldsymbol{E}^{(1)}, \boldsymbol{H}^{(1)} ; \boldsymbol{E}^{(2)}, \boldsymbol{H}^{(2)}$ fulfils the system (2.1) and will be called a modal field (polarization mode). Formula (2.10) gives the modal decomposition (cp. Bohren's decomposition [1]). Functions $u, v$ will be referred to as modal potentials.

Plane wave solutions to (2.1) follow easily from (2.8a,b) via (2.11) and (2.12), their circular polarization may be easily checked.

\section{Formulation of the problem}

For the chiral medium (1.1) we assume $\varepsilon, \mu$ to be real positive, $\chi$ to be real and $|\chi|<\sqrt{\varepsilon \mu}$. Thus the medium is lossless and of the conventional type. Small losses may be temporarily admitted to aid in some considerations but are dropped afterwards.

The incident plane wave can be of either mode and is given via (2.11) by

$$
u_{i}=a \mathrm{e}^{\mathrm{i} k_{1} s^{(1)} \cdot \rho}=a \mathrm{e}^{\mathrm{i}\left(\alpha_{10} x+\gamma_{10} z\right)}
$$

or via (2.12) by

$$
\begin{aligned}
& v_{i}=b \mathrm{e}^{\mathrm{i} k_{2} s^{(2)} \cdot \rho}=b \mathrm{e}^{\mathrm{i}\left(\alpha_{20} x+\gamma_{20} z\right)} \\
& \rho=x x+y y, \quad k_{n} s^{(n)}=\alpha_{n 0} x+\gamma_{n 0} z, \quad \alpha_{n 0}^{2}+\gamma_{n 0}^{2}=k_{n}^{2}, \\
& \alpha_{n 0}^{2}<k_{n}^{2}, \quad \gamma_{n 0}>0, \quad n=1,2 .
\end{aligned}
$$

Both waves (3.1), (3.2) will be considered simultaneously; only in the final results we shall assume $b=0$ or $a=0$.

In order to find the solution we shall seek the modal potentials $u, v$ corresponding to the scattered field $\boldsymbol{E}_{\mathbf{s}}, \boldsymbol{H}_{\mathbf{s}}$.

The functions $u, v$ will be determined uniquely by the following stipulations: 1. equations -

respectively $(2.8 \mathrm{a}, \mathrm{b})$

2. boundary conditions -

$$
E_{y s}=u+v=-\left(u_{i}+v_{i}\right)
$$


for $z=0, x \geq 0$;

$$
k_{1} k_{2} E_{x \mathrm{~s}}=\frac{\partial}{\partial z}\left(k_{2} u-k_{1} v\right)=-\frac{\partial}{\partial z}\left(k_{2} u_{i}-k_{1} v_{i}\right)
$$

3. edge conditions -

$$
u=c_{1}+\mathrm{O}\left(\rho^{1 / 2}\right), \quad v=c_{2}+\mathrm{O}\left(\rho^{1 / 2}\right) \text { for } \rho=\sqrt{x^{2}+z^{2}} \rightarrow 0
$$

$c_{1}, c_{2}$ are some constants;

4. outgoing wave conditions -

$$
u-u_{\mathrm{g}}, \quad v-v_{\mathrm{g}}
$$

decay exponentially for $\rho \rightarrow \infty$ if small losses are admitted, $u_{\mathrm{g}}, v_{\mathrm{g}}$ denote the solutions for the problem, determined by geometrical optics. and (2.9).

Conditions (3.5) follow from similar conditions for $E_{y \mathrm{~s}}$ and $H_{y \mathrm{~s}}$ via (2.7)

\section{Even and odd parts of the problem}

We now decompose the boundary value problem of Sec. 3 into its even and odd parts with respect to $z$. Thus

where

$$
u=u^{(\mathrm{e})}+u^{(\mathrm{o})}, \quad v=v^{(\mathrm{e})}+v^{(\mathrm{o})},
$$

$$
\begin{array}{ll}
u^{(\mathrm{e})}(x,-z)=u^{(\mathrm{e})}(x, z), & v^{(\mathrm{e})}(x,-z)=v^{(\mathrm{e})}(x, z), \\
u^{(\circ)}(x,-z)=-u^{(\circ)}(x, z), & v^{(\circ)}(x,-z)=-v^{(\circ)}(x, z) .
\end{array}
$$

For the even parts $u^{(\mathrm{e})}, v^{(\mathrm{e})}$ we require at the plane $z=0$ the following boundary conditions to be satisfied:

for $x<0$

for $x>0$

$$
\frac{\partial u^{(e)}}{\partial z}=0, \quad \frac{\partial v^{(e)}}{\partial z}=0
$$

$$
u^{(\mathrm{e})}+v^{(\mathrm{e})}=-a \mathrm{e}^{\mathrm{i} \alpha_{10} x}-b \mathrm{e}^{\mathrm{i} \alpha_{20} x}, \quad \frac{\partial}{\partial z}\left(k_{2} u^{(\mathrm{e})}-k_{1} v^{(\mathrm{e})}\right)=0 .
$$

For the odd part we require:

for $x<0$

for $x>0$

$$
u^{(0)}=0, \quad v^{(0)}=0,
$$

$$
u^{(\circ)}+v^{(\circ)}=0, \quad \frac{\partial}{\partial z}\left(k_{2} u-k_{1} v\right)=-a \mathrm{i} k_{2} \gamma_{10} \mathrm{e}^{\mathrm{i} \alpha_{10} x}+\mathrm{i} b k_{1} \gamma_{20} \mathrm{e}^{\mathrm{i} \alpha_{20} x}
$$

The remaining uniqueness conditions for the pairs $u^{(\mathrm{e})}, v^{(\mathrm{e})}$ and $u^{(\circ)}, v^{(\circ)}$ are the same as those for $u, v$ set in Sec. 3 .

Let us assume that even and odd solutions have been found for $z>0$ and that they are determined for $z<0$ by (4.2) and (4.3). Then $u, v$ given by (4.1) are continuous along with their normal derivatives across the aperture $x<0$ and consequently fulfil $(2.8 \mathrm{a}, \mathrm{b})$ everywhere outside the half-plane. Since $\frac{\partial}{\partial z}\left(u^{(\mathrm{e})}+v^{(\mathrm{e})}\right)$ and $u^{(\circ)}+v^{(\circ)}$ are continuous across the half-plane (despite possible discontinuities in $\left.\partial u^{(\mathrm{e})} / \partial z, \partial v^{(\mathrm{e})} / \partial z, u^{(\circ)}, v^{(\circ)}\right)$, thus the boundary conditions (3.4) are also satisfied and consequently a solution for the problem of Sec. 3 is obtained. 


\section{Solution for the even part}

We shall consider the half-space $z \geq 0$ and assume there the following plane-wave spectral representations for $u^{(\mathrm{e})}, v^{(\mathrm{e})}$ :

$$
u^{(\mathrm{e})}=\int_{Q} A^{(\mathrm{e})}(\alpha)^{\mathrm{i}\left(\alpha x+\gamma_{1} z\right)} \mathrm{d} \alpha, \quad v^{(\mathrm{e})}=\int_{Q} B^{(\mathrm{e})} \mathrm{e}^{\mathrm{i}\left(\alpha x+\gamma_{2} z\right)} \mathrm{d} \alpha .
$$

Integration in (5.1) is in the complex $\alpha$-plane, $A^{(\mathrm{e})}(\alpha)=A^{(\mathrm{e})}, B^{(\mathrm{e})}(\alpha)=B^{(\mathrm{e})}$ are the unknown amplitude functions to be found, $\gamma_{n}(\alpha)=\gamma_{n}=\sqrt{k_{n}^{2}-\alpha^{2}}$, $n=1,2$. The contour $Q$ follows the real axis except for appropriate indentations such that $-k_{n}$ are below $Q$ and $\alpha_{n 0}, k_{n}$ are above $Q$ as shown in Fig. 2 where also the branch cuts $\Gamma_{+}^{(n)}, \Gamma_{-}^{(n)}$ outgoing from $\pm k_{n}$ respectively are indicated. We choose the roots $\gamma_{n}$ so that for $\alpha=0, \gamma_{n}=k_{n}$. We denote by $\Omega_{U}$ the region above $Q$ and by $\Omega_{L}$ that below $Q$.

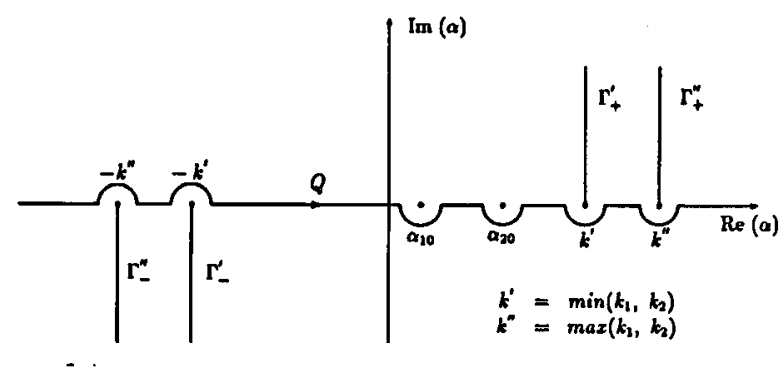

Fig. 2. The complex $\alpha$-plane with the contour $Q$, the branch points $\pm k_{1}, \pm k_{2}$, the branch cuts $\Gamma_{ \pm}^{(1)}, \Gamma_{ \pm}^{(2)}$ and the incident wave poles $\alpha_{10}, \alpha_{20}$.

The validity of representations of form (5.1) depends on the convergence of the integrals. This will be verified after the solution has been constructed. If the integrals converge, conditions (3.3) will be fulfilled. Since for a slightly lossy medium $\gamma_{n}$ have small positive imaginary parts, it can be show that conditions (3.6) are also satisfied.

We shall now deal with the boundary and edge conditions (4.4), (4.5) and (3.5) by requiring some combinations of $A^{(\mathrm{e})}, B^{(\mathrm{e})}$ to have certain analytical properties and an appropriate behaviour for $|\alpha| \rightarrow \infty$.

Consider (5.1) for $z=0$. In view of (4.4) the condition (4.5b) is fulfilled for $-\infty<x<\infty$, consequently

$$
k_{2} \gamma_{1} A^{(\mathrm{e})}-k_{1} \gamma_{2} B^{(\mathrm{e})}=0 \text {. }
$$

Condition (4.5a) will be satisfied if

$$
A^{(\mathrm{e})}+B^{(\mathrm{e})}=U(\alpha)-\frac{1}{2 \pi \mathrm{i}}\left(\frac{a}{\alpha-\alpha_{10}}+\frac{b}{\alpha-\alpha_{20}}\right),
$$

where $U(\alpha)$ is analytic in $\Omega_{U}+Q$ and behaves properly for $|\alpha| \rightarrow \infty$.

In order to satisfy the edge conditions (3.5), $U(\alpha)$ is required to have the following asymptotic behaviour [2]:

$$
U(\alpha)-\frac{1}{2 \pi \mathrm{i}}\left(\frac{a}{\alpha-\alpha_{10}}+\frac{b}{\alpha-\alpha_{20}}\right)=\mathrm{O}\left(\alpha^{-3 / 2}\right) \text { for }|\alpha| \rightarrow \infty \text { in } \Omega_{U}+Q
$$


The condition (4.4a) implies

$\gamma_{1} A^{(e)}=k_{1} L(\alpha)$,

where $L(\alpha)$ is analytic in $\Omega_{L}+Q$ and does not grow too fast for $|\alpha| \rightarrow \infty\left(k_{1}\right.$ in (5.4) is introduced for the sake of symmetry).

From $(5.2 \mathrm{a}, \mathrm{b})$ and $(5.4)$ we obtain the Wiener-Hopf equation to be solved

$U(\alpha)-\frac{1}{2 \pi \mathrm{i}}\left(\frac{a}{\alpha-\alpha_{10}}+\frac{b}{\alpha-\alpha_{20}}\right)=G(\alpha) L(\alpha)$,

where

$$
G(\alpha)=G=\frac{g(\alpha)}{\gamma_{1} \gamma_{2}}, \quad g(\alpha)=k_{2} \gamma_{1}+k_{1} \gamma_{2} .
$$

For the chosen roots of $\gamma_{n}$ we have $g(\alpha) \neq 0$ in the whole $\alpha$ plane cut by $\Gamma_{ \pm}^{(n)}$.

Let us factor $g(\alpha)$ in the Wiener-Hopf sense

$$
g(\alpha)=g_{U}(\alpha) g_{L}(\alpha)
$$

where $g_{U}(\alpha)=g_{U}$ is analytic in $\Omega_{U}+Q$ and $g_{L}(\alpha)=g_{L}$ is analytic in $\Omega_{L}+Q$. We have [2]:

$$
g_{U}(\alpha)=\exp \left(\frac{1}{2 \pi \mathrm{i}} \int_{Q} \frac{\log g(t)}{t-\alpha} \mathrm{d} t\right), \quad g_{L}(\alpha)=g_{U}(-\alpha),
$$

Effective evaluation of (5.7) is possible [3, 4], but is postponed to a later paper concerned with an investigation and interpretation of the present solution.

From (5.6) and (5.7b) we get

$g_{U}(\alpha)=\mathrm{O}\left(\alpha^{1 / 2}\right)$ for $|\alpha| \rightarrow \infty$.

Factorization of $\gamma_{1}(\alpha) \cdot \gamma_{2}(\alpha)$ is straightforward and consequently

$$
G(\alpha)=G_{U}(\alpha) G_{L}(\alpha),
$$

where

$$
G_{U}(\alpha)=G_{U}=\frac{g_{U}(\alpha)}{\sqrt{\left(k_{1}+\alpha\right)\left(k_{2}+\alpha\right)}}, \quad G_{L}(\alpha)=G_{L}=\frac{g_{L}(\alpha)}{\sqrt{\left(k_{1}-\alpha\right)\left(k_{2}-\alpha\right)}} .
$$

$G_{U}$ is analytic in $\Omega_{U}+Q$ and $G_{L}$ in $\Omega_{L}+Q$ moreover

$$
G_{U}(\alpha)=\mathrm{O}\left(\alpha^{-1 / 2}\right) \text { for }|\alpha| \rightarrow \infty \text {. }
$$

From (5.5) and (5.9) we obtain

$$
G_{L}(\alpha) L(\alpha)=G_{U}^{-1}(\alpha)\left[U(\alpha)-\frac{1}{2 \pi \mathrm{i}}\left(\frac{a}{\alpha-\alpha_{10}}+\frac{b}{\alpha-\alpha_{20}}\right)\right] .
$$

Let us rewrite (5.11) in the following form:

$$
\begin{aligned}
& G_{L}(\alpha) L(\alpha)+\frac{1}{2 \pi \mathrm{i}}\left(\frac{G_{U}^{-1}\left(\alpha_{10}\right) a}{\alpha-\alpha_{10}}+\frac{G_{U}^{-1}\left(\alpha_{20}\right) b}{\alpha-\alpha_{20}}\right)=G_{U}^{-1}(\alpha) U(\alpha) \\
& -\frac{1}{2 \pi \mathrm{i}} \frac{\left[G_{U}^{-1}(\alpha)-G_{U}^{-1}\left(\alpha_{10}\right)\right] a}{\alpha-\alpha_{10}}-\frac{1}{2 \pi \mathrm{i}} \frac{\left[G_{U}^{-1}(\alpha)-G_{U}^{-1}\left(\alpha_{20}\right)\right] b}{\alpha-\alpha_{20}} .
\end{aligned}
$$


The left-hand side of (5.12) is analytic in $\Omega_{L}+Q$ and the right-hand side is analytic in $\Omega_{U}+Q$, thus (5.12) is equal to an entire function. However in view of (5.3) and (5.10) this entire function must be zero and consequently we get from (5.12):

$$
\begin{aligned}
U(\alpha) & =\frac{1}{2 \pi \mathrm{i}}\left(\frac{a}{\alpha-\alpha_{10}}+\frac{b}{\alpha-\alpha_{20}}\right) \\
& -\frac{G_{U}(\alpha) G_{U}^{-1}\left(\alpha_{10}\right) a}{2 \pi \mathrm{i}\left(\alpha-\alpha_{10}\right)}-\frac{G_{U}(\alpha) G_{U}^{-1}\left(\alpha_{20}\right) b}{2 \pi \mathrm{i}\left(\alpha-\alpha_{20}\right)} .
\end{aligned}
$$

Thus, via $(5.2 \mathrm{a}, \mathrm{b})$ we obtain

$$
\begin{aligned}
& A^{(\mathrm{e})}(\alpha)=\frac{-k_{1}}{2 \pi \mathrm{i}} \sqrt{\frac{k_{2}-\alpha}{k_{1}+\alpha}} g_{L}^{-1}(\alpha)\left[\frac{G_{U}^{-1}\left(\alpha_{10}\right) a}{\alpha-\alpha_{10}}+\frac{G_{U}^{-1}\left(\alpha_{20}\right) b}{\alpha-\alpha_{20}}\right], \\
& B^{(\mathrm{e})}(\alpha)=\frac{k_{2}}{k_{1}} \frac{\gamma_{1}}{\gamma_{2}} A^{(\mathrm{e})} .
\end{aligned}
$$

From (5.14a,b), (5.7b), (5.8), (5.10) we easily verify that integrals in (5) converge.

\section{Solution for the odd part}

As in previous section, we assume for $z \geq 0$ the following representations:

$$
u^{(\circ)}=\int_{Q} A^{(\circ)}(\alpha) \mathrm{e}^{\mathrm{i}\left(\alpha x+\gamma_{1} z\right)} \mathrm{d} \alpha, \quad v^{(\circ)}=\int_{Q} B^{(\circ)}(\alpha) \mathrm{e}^{\mathrm{i}\left(\alpha x+\gamma_{2} z\right)} \mathrm{d} \alpha .
$$

$A^{(0)}(\alpha)=A^{(0)}, B^{(0)}(\alpha)=B^{(0)}$ are the amplitude functions to be found. The remaining notation and remarks are the same as for the even part.

From (4.6) and (4.7a) we get

$$
A^{(0)}+B^{(0)}=0 \text {. }
$$

From (4.7b) we have

$$
k_{2} \gamma_{1} A^{(\circ)}-k_{1} \gamma_{2} B^{(\circ)}=U^{\prime}(\alpha)-\frac{1}{2 \pi \mathrm{i}}\left(\frac{k_{2} \gamma_{10} a}{\alpha-\alpha_{10}}-\frac{k_{1} \gamma_{20} b}{\alpha-\alpha_{20}}\right),
$$

where $U^{\prime}(\alpha)$ is to be analytic in $\Omega_{U}+Q$ and by virtue of the edge conditions (3.5) is required to have the following asymptotic behaviour:

$$
U^{\prime}(\alpha)-\frac{1}{2 \pi \mathrm{i}}\left(\frac{k_{2} \gamma_{10} a}{\alpha-\alpha_{10}}-\frac{k_{1} \gamma_{20} b}{\alpha-\alpha_{20}}\right)=\mathrm{O}\left(\alpha^{-1 / 2}\right) \text { for }|\alpha| \rightarrow \infty .
$$

The condition (4.6a) implies

$$
A^{(\mathrm{o})}=L^{\prime}(\alpha)
$$

where $L^{\prime}(\alpha)$ is to be analytic in $\Omega_{L}+Q$ and behave properly at infinity.

From (6.2), (6.4) we obtain

$$
U^{\prime}(\alpha)-\frac{1}{2 \pi \mathrm{i}}\left(\frac{k_{2} \gamma_{10} a}{\alpha-\alpha_{10}}-\frac{k_{1} \gamma_{20} b}{\alpha-\alpha_{20}}\right)=g(\alpha) L^{\prime}(\alpha) .
$$

In the way identical as in Sec. 5 we obtain

$$
U^{\prime}(\alpha)=\frac{k_{1} \gamma_{10}\left[1-g_{U}(\alpha) g_{U}^{-1}\left(\alpha_{10}\right)\right] a}{2 \pi \mathrm{i}\left(\alpha-\alpha_{10}\right)}-\frac{k_{1} \gamma_{20}\left[1-g_{U}(\alpha) g_{U}^{-1}\left(\alpha_{20}\right)\right] b}{2 \pi \mathrm{i}\left(\alpha-\alpha_{10}\right)}
$$


This, via (6.2) gives

$$
\begin{aligned}
& A^{(\circ)}=-\frac{1}{2 \pi \mathrm{i} g_{L}(\alpha)}\left[\frac{k_{2} \gamma_{10} g_{U}^{-1}\left(\alpha_{10}\right) a}{\alpha-\alpha_{10}}-\frac{k_{1} \gamma_{20} g_{U}^{-1}\left(\alpha_{20}\right) b}{\alpha-\alpha_{10}}\right], \\
& B^{(\circ)}=-A^{\circ}(\alpha) .
\end{aligned}
$$

Convergence of the integrals is easily verified.

\section{Complete solution}

Collecting the formulas (4.1), (4.2), (4.3), (5.1), (6.1) we obtain the following solution for the problem formulated in Sec. 3:

$$
u=\int_{Q} A^{( \pm)}(\alpha) \mathrm{e}^{\mathrm{i}\left(\alpha x \pm \gamma_{1} z\right)} \mathrm{d} \alpha, \quad v=\int_{Q} B^{( \pm)}(\alpha) \mathrm{e}^{\mathrm{i}\left(\alpha x \pm \gamma_{2} z\right)} \mathrm{d} \alpha,
$$

where

$$
A^{( \pm)}(\alpha)=A^{(\mathrm{e})}(\alpha) \pm A^{(\circ)}(\alpha), \quad B^{( \pm)}(\alpha)=B^{(\mathrm{e})}(\alpha) \pm B^{(\circ)}(\alpha),
$$

the upper signs are for $z \geq 0$ and the lower are for $z \leq 0$.

The decomposition (4.1) remains useful in analytical investigations of the solution (7.1).

\section{Reduction to an ordinary, isotropic medium}

For $\chi=0$ we get $k_{1}=k_{2}=k, \gamma_{1}=\gamma_{2}=\gamma$ and the solutions (5.14), (6.7), (7.2) reduce correctly to those for an ordinary, isotropic medium. Since however each of the incident waves (3.1), (3.2) defines electromagnetic waves of circular polarization, we obtain (even for only one incident wave) linear combinations of conventional solutions constructed for linearly polarized incident waves defined either by $E_{y}$ or $H_{y}$.

In order to get these conventional solutions, we shall consider the components $E_{y s}, H_{y s}$ defined by (2.1) and (2.9):

$$
E_{y \mathrm{~s}}=u+v, \quad H_{y \mathrm{~s}}=-\mathrm{i} Y(u-v) .
$$

We assume in (3.1), (3.2) that $\alpha_{10}=\alpha_{20}=\alpha_{0}$.

Consider the case $a=b$, then

$$
A^{(\mathrm{e})}=B^{(\mathrm{e})}=-\sqrt{\frac{k+\alpha_{0}}{k+\alpha}} \frac{a}{2 \pi \mathrm{i}\left(\alpha-\alpha_{0}\right)}, \quad A^{(\circ)}=-B^{(\circ)}=0 .
$$

This via (7.2) coincides with a direct solution for $E_{y}$ polarization.

For $a=-b$ we get

$$
A^{(\mathrm{e})}=B^{(\mathrm{e})}=0, \quad A^{(\mathrm{o})}=-B^{(\circ)}=-\sqrt{\frac{k+\alpha_{0}}{k-\alpha}} \frac{a}{2 \pi \mathrm{i}\left(\alpha-\alpha_{0}\right)},
$$

and this also agrees with a direct solution for $H_{y}$ polarization. 


\section{Properties of the solution}

Analysis of the solution concerns its many properties and involves several viewpoints as evidenced by [3, 4, 8-11]. A crucial simplification results from an effective factorization of $g(\alpha)[3,4]$. A detailed investigation and interpretation is postponed to a later paper, here we only outline some of the basic properties of the solution.

\subsection{Far field}

Formal expressions for the far fields can be extracted from (7.2). Assuming the singularities at $\alpha_{n_{0}}, \pm k_{n}, n=1,2$ to be well separated, we deform $Q$ into the steepest descents path and, substituting $x=\rho \cos \theta, z=\rho \sin \theta$, we get

$$
\begin{aligned}
& u_{\mathrm{f}}=\sqrt{2 \pi k_{1}} A^{( \pm)}\left(k_{1} \cos \theta\right) \frac{\sin \theta \mathrm{e}^{\mathrm{i} k_{1} \rho}}{\sqrt{\rho}}+\mathrm{O}\left(\rho^{-3 / 2}\right), \\
& v_{\mathrm{f}}=\sqrt{2 \pi k_{2}} B^{( \pm)}\left(k_{2} \cos \theta\right) \frac{\sin \theta \mathrm{e}^{\mathrm{i} k_{2} \rho}}{\sqrt{\rho}}+\mathrm{O}\left(\rho^{-3 / 2}\right) .
\end{aligned}
$$

Even for only one incident wave, two families of diffracted rays appear in (9.1), thereby illustrating the modal coupling.

For one incident wave, say $b=0$, formulas (9.1) can be rephrased in the following way:

$$
u_{\mathrm{f}}=D_{11}\left(\theta, \alpha_{10}\right) a \frac{\mathrm{e}^{\mathrm{i} k_{1} \rho}}{\sqrt{\rho}}, \quad v_{\mathrm{f}}=D_{12}\left(\theta, \alpha_{10}\right) a \frac{\mathrm{e}^{\mathrm{i} k_{2 \rho} \rho}}{\sqrt{\rho}}
$$

where $D_{11}, D_{12}$ are the diffraction coefficients in the sense of Keller's geometrical theory of diffraction. For $a=0$ we get the coefficients $D_{22}\left(\theta, \alpha_{20}\right), D_{21}\left(\theta, \alpha_{20}\right)$ and a matrix $D$ of the diffraction coefficients can be formed. Obviously the potentials $u, v$ can be eliminated and results analogous to (9.1), (9.2) given directly for the electromagnetic field with an appropriate tensor of diffraction coefficients.

\subsection{Lateral waves}

While deforming the contour $Q$ into the steepest descents paths we encounter the "alien" branch cuts $\Gamma_{ \pm}^{(2)}$ for $u$ and $\Gamma_{ \pm}^{(1)}$ for $v$. Contributions from these cuts determine lateral waves propagating along the half-plane and existing in appropriate sectors of $\theta$. Consider the case $\chi>0$, then $k_{1}>k_{2}$ and we get

$$
\begin{aligned}
& u_{l}^{( \pm)}=\frac{a_{l}^{( \pm)}(\theta)}{\rho^{3 / 2}} \mathrm{e}^{\mathrm{i}\left(k_{2} x \pm \tau z\right)}+\mathrm{O}\left(\rho^{-5 / 2}\right), \\
& v_{l}^{( \pm)}=\frac{b_{l}^{( \pm)}(\theta)}{\rho^{3 / 2}} \mathrm{e}^{\mathrm{i}\left(k_{1} x \pm \mathrm{i} \tau z\right)}+\mathrm{O}\left(\rho^{-5 / 2}\right),
\end{aligned}
$$

where $\tau=2(\chi \sqrt{\varepsilon \mu})^{1 / 2}$, the upper sign is for $z>0$ and the lower is for $z<0$. Amplitudes $a_{l}^{( \pm)}(\theta), b_{l}^{( \pm)}(\theta)$ and the sectors where the waves exist can be determined in the way described in $[4,8,10]$.

The waves (9.3a) can be interpreted in terms of geometrical optics and, in particular, directions of their rays are easy to determine. It would be interesting to 
observe experimentally the shadows cast by the lateral wave (9.3a) in the shadow region of the incident waves $u_{i}, v_{i}$. Interpretation of $(9.3 \mathrm{~b})$ is more complicated and requires introduction of complex rays.

\subsection{Geometrical optics field}

Consider the case $b=0$, then for $z \geq 0$ and the angle of observation $\theta<\theta_{10}=\arccos \left(\alpha_{10} / k_{1}\right)$, the pole at $\alpha=\alpha_{10}$ will be crossed on deforming the contour $Q$ and by evaluating the respective residua in (7.1) we easily obtain

$$
u=-u_{\mathrm{i}}+\mathrm{O}\left(\rho^{-1 / 2}\right), \quad v=\mathrm{O}\left(\rho^{-1 / 2}\right), \quad \rho \rightarrow \infty .
$$

For $z \leq 0$ and $\theta>2 \pi-\theta_{10}$ we get

$$
u=\frac{k_{2} \gamma_{10}-k_{1} \gamma_{20}\left(\alpha_{10}\right)}{g\left(\alpha_{10}\right)} \mathrm{e}^{\mathrm{i}\left(\alpha_{10} x-\alpha_{10} z\right)}+\mathrm{O}\left(\rho^{-1 / 2}\right),
$$

for $\theta>2 \pi-\theta_{12}, \theta_{12}=\arccos \left(\alpha_{10} / k_{2}\right)$ we have

$$
v=\frac{-2 k_{2} \gamma_{10}}{g\left(\alpha_{10}\right)} \mathrm{e}^{\mathrm{i}\left[\alpha_{10} x-\gamma_{2}\left(\alpha_{10}\right) z\right]}+\mathrm{O}\left(\rho^{-1 / 2}\right) .
$$

These results agree with $u_{\mathrm{g}}, v_{\mathrm{g}}$ evaluated directly according to geometrical optics and exhibit clearly the presence of reflection coupling. Similar results follow for $a=0$.

\subsection{Edge behaviour}

It is interesting to observe that the behaviour of an electromagnetic field at an edge is in chiral media the same as in ordinary, isotropic ones.

By virtue of (5.8), from (5.13), (5.2), and (6.6), (6.2) we get

$$
u=p_{1}(x)+c_{1}^{\prime} x^{1 / 2}+\mathrm{O}\left(x^{3 / 2}\right), \quad v=p_{2}(x)+c_{2}^{\prime} x^{1 / 2}+\mathrm{O}\left(x^{3 / 2}\right) \quad(9.6 \mathrm{a}, \mathrm{b})
$$
for $z=0, x \rightarrow 0 ; p_{1}(x), p_{2}(x)$ are regular functions, $c_{1}^{\prime}, c_{2}^{\prime}$ are constants. The field behaviour is given via (2.11), (2.12).

\section{Conclusions}

An exact, closed form solution has been presented for a new, electromagnetic, half-plane diffraction problem whose fundamental features are:

1. a two-mode character comprising reflection coupling;

2. interaction of two wave species resulting in the excitation of lateral waves.

It is likely that this is the simplest problem characterized by points (1) and (2). Thus analysis of these properties and of their physical significance can be conducted in the simple situation of the present case. For oblique incidence, the structure of the solution, to be presented in a forthcoming paper [12], is technically much more complicated; however its core is almost identical with the present problem.

Within electrodynamics of chiral media this is the first example of an edge diffraction problem in an unbounded chiral medium. Exact solutions for diffraction by a chiral cylinder and a chiral sphere embedded in another chiral medium were constructed earlier. 


\section{Appendix}

If $\left[\left(\nabla^{2}+k_{1}^{2}\right)\left(\nabla^{2}+k_{2}^{2}\right) \phi=0 ; k_{1} \neq k_{2}\right]$ then $\left[\phi=u+v,\left(\nabla^{2}+k_{1}^{2}\right) u=0\right.$, $\left.\left(\nabla^{2}+k_{2}^{2}\right) v=0\right]$.

Proof: $\left(\nabla^{2}+k_{1}^{2}\right)\left(\nabla^{2}+k_{2}^{2}\right) \phi=0,\left(\nabla^{2}+k_{2}^{2}\right) \phi=u^{\prime},\left(\nabla^{2}+k_{1}^{2}\right) u^{\prime}=0$, let $\phi=v+u^{\prime \prime},\left(\nabla^{2}+k_{2}^{2}\right) v=0,\left(\nabla^{2}+k_{2}^{2}\right) u^{\prime \prime}=u^{\prime}$. Assume $u^{\prime \prime}=q u^{\prime}=u$ then $\left(\nabla^{2}+k_{2}^{2}\right) u^{\prime \prime}=u^{\prime}$ if $q=\left(k_{2}^{2}-k_{1}^{2}\right)^{-1}$ and $\left(\nabla^{2}+k_{1}^{2}\right) u=0$. Thus $\phi=u+v$ q.e.d.

\section{References}

[1] A. Lakhtakia, V.K. Varadan, V.V. Varadan,Time-Harmonic Electromagnetic Fields in Chiral Media, Springer-Verlag, Berlin 1989.

[2] B. Noble, Methods Based on the Wiener-Hopf Technique, Pergamon Press, London 1958.

[3] W.S. Ament, Application of a Wiener-IIopf Technique to Certain Diffraclion Problems, U.S. Na val Res. Lab. Report No 4334, 1954.

[4] H. Stöckel, Ann. der Phys. 7, 156 (1963).

[5] T.B.A. Senior, Appl. Sci. Res. B 8, 35 (1960).

[6] S. Przeździecki, J. Appl. Phys. 37, 2768 (1966).

[7] R.A. Hurd, S. Przeździecki, IEEE Trans. Anlennas Propag. AP-33, 813 (1985).

[8] B. Jaskorzyńska-Dzieciaszek, Ph.D. Thesis, Inst. Fund. Technolog. Res., Polish Academy of Sciences, Warszawa 1981.

[9] A. Ciarkowski, Wave Molion, to appear.

[10] II.W.M. Witlox, M.Sc. Thesis, Eindhoven Univ. of Techn., Eindhoven 1983.

[11] R.D. Coblin, L.W. Pearson, Radio Sci. 19, 1277 (1984).

[12] S. Przeździecki, to be published. 